\title{
Domain Engineering for Enhanced Ferroelectric Properties of Epitaxial (001) BiFeO Thin Films
}

\author{
By Ho Won Jang, Daniel Ortiz, Seung-Hyub Baek, Chad M. Folkman, \\ Rasmi R. Das, Padraic Shafer, Yanbin Chen, Christofer T. Nelson, \\ Xiaoqing Pan, Ramamoorthy Ramesh, and Chang-Beom Eom*
}

Multiferroic $\mathrm{BiFeO}_{3}$ has attracted great interest due to its promising application to magnetoelectric devices. ${ }^{[1-3]}$ In addition, the high remanent polarization and piezoelectric response of $\mathrm{BiFeO}_{3}$ thin films, which are comparable to those of conventional Ti-rich lead zirconia titanate, suggested $\mathrm{BiFeO}_{3}$ as a strong candidate for lead-free nonvolatile memories. ${ }^{[4]} \mathrm{BiFeO}_{3}$ has a rhombohedral perovskite structure with pseudocubic lattice parameters $a_{\mathrm{r}}=3.96 \AA$ and $\alpha_{\mathrm{r}}=0.6^{\circ}$. $^{[5]}$ Due to this low symmetry, (001)-oriented epitaxial $\mathrm{BiFeO}_{3}$ films possess the rhombohedral distortion along one of the four (111) crystallographic directions of the pseudocubic perovskite unit cell. ${ }^{[6]}$ Thus, eight possible polarization (ferroelectric) variants, which correspond to four structural (ferroelastic) domains, may form in the films, leading to complex domain patterns with both $\{100\}$ and $\{101\}$ twin boundaries. ${ }^{[6,7]}$ Such a complex domain structure can deteriorate the ferroelectric response of the system by external electric field, and complicates the examination of the coupling between magnetic and ferroelectric order parameters in $\mathrm{BiFeO}_{3}{ }^{[3]}$ Recently, several studies have shown that high-quality (001)oriented $\mathrm{BiFeO}_{3}$ thin films with two-variant stripe domains can be achieved by using miscut ${ }^{[8]}$ and orthorhombic ${ }^{[9,10]}$ substrates. However, the clear identification of the origin of the stripe domains in $\mathrm{BiFeO}_{3}$ thin films has not yet been reported. Furthermore, correlating the ferroelastic domain structures of $\mathrm{BeFiO}_{3}$ thin films with the ferroelectric properties is a critical, yet missing link between materials properties and device performance.

In this communication, we report the origin of the ferroelastic domain variant selection in (001) $\mathrm{BiFeO}_{3}$ films on miscut (001) $\mathrm{SrTiO}_{3}$ substrates with coherent $\mathrm{SrRuO}_{3}$ bottom electrodes, and its effect on the ferroelectric properties of the films. To our best

[*] Prof. C. B. Eom, Dr. H. W. Jang, D. Ortiz, S. H. Baek, C. M. Folkman, R. R. Das

Department of Materials Science and Engineering

University of Wisconsin

Madison, WI 53706 (USA)

E-mail: eom@engr.wisc.edu

P. Shafer, Prof. R. Ramesh

Department of Physics and Department of Materials Science and Engineering

University of California

Berkeley, CA 94720 (USA)

Dr. Y. B. Chen, C. T. Nelson, Prof. X. Q. Pan

Department of Materials Science and Engineering

University of Michigan

Ann Arbor, MI 48109 (USA)

DOI: 10.1002/adma.200800823 knowledge, this is the first report that ferroelectric switching behavior and leakage current in $\mathrm{BiFeO}_{3}$ films are simultaneously improved by domain engineering. For the demonstration of the domain variant selection in $\mathrm{BiFeO}_{3}$ films by substrate miscut, the $\mathrm{SrTiO}_{3}$ substrate was chosen to have either 0.05 or $4^{\circ}$ miscut toward the [100] direction, which corresponds to the downhill miscut direction. Since the step width of the $0.05^{\circ}$ miscut substrate $(\sim 460 \mathrm{~nm})$ is much larger than that of the $4^{\circ}$ miscut substrate $(6 \mathrm{~nm})$, the effects of the substrate on the strain relaxation and domain structure can be resolved using both substrates (in this letter we call $0.05^{\circ}$ miscut exact and $4^{\circ}$ miscut miscut, for convenience). Atomic force microscopy (AFM) and reciprocal space mapping (RSM) using high-resolution X-ray diffraction (HRXRD) show that the miscut substrate leads to stepflow growth and two-variant strip domains in the $\mathrm{BiFeO}_{3}$ film. In contrast, the exact substrate causes 3D island-growth and fourvariant domains. Combined with transmission electron microscopy (TEM) and piezoelectric force microscopy (PFM) results, it is suggested that both the preferential distortion of unit cells and the complete step-flow growth induced by the substrate anisotropy are the origins of the formation of the two-variant stripe domains in (001) $\mathrm{BiFeO}_{3}$ films. Finally, the polarizationelectric field $(P-E)$ hysteresis loop and leakage-current measurements allow us to find that two-variant stripe domains provide complete ferroelectric switching in $\mathrm{BiFeO}_{3}$ thin films with low leakage current.

The growth mode of the epitaxial $\mathrm{BiFeO}_{3}$ film can be clarified by monitoring the change of surface morphology with increasing film thickness. AFM images were obtained before and after the deposition of $\mathrm{BiFeO}_{3}$ films on both exact and miscut (001) $\mathrm{SrTiO}_{3}$ substrates with coherent $\mathrm{SrRuO}_{3}$ bottom electrodes, as shown in Figure 1 . The surface of the $100 \mathrm{~nm}$ thick $\mathrm{SrRuO}_{3}$ on the exact substrate is atomically smooth with one unit cell high (4®) steps (Fig. 1a). The terrace width is $\sim 500 \mathrm{~nm}$, which is consistent with the miscut angle of $0.05^{\circ}$. After the deposition of $\mathrm{BiFeO}_{3}$ on top of $\mathrm{SrRuO}_{3}$ bottom electrode, the $100 \mathrm{~nm}$ thick $\mathrm{BiFeO}_{3}$ film on the exact substrate exhibits many protrusions and holes (Fig. 1b). The propagation of atomic steps is randomly oriented, as indicated by white arrows. With increasing thickness to $400 \mathrm{~nm}$, the film shows a much rougher surface with big islands (Fig. 1c), consistent with the 3D islandformation growth mode. ${ }^{[11]}$

In contrast, completely different surface morphology can be observed in $\mathrm{BiFeO}_{3}$ films on the miscut substrate. The AFM image of the $\mathrm{SrRuO}_{3}$ bottom electrode shows the periodic steps (Fig. 1d), originating from step bunching due to the small terrace width. The actual terrace width $(140 \mathrm{~nm})$ is much larger than the 

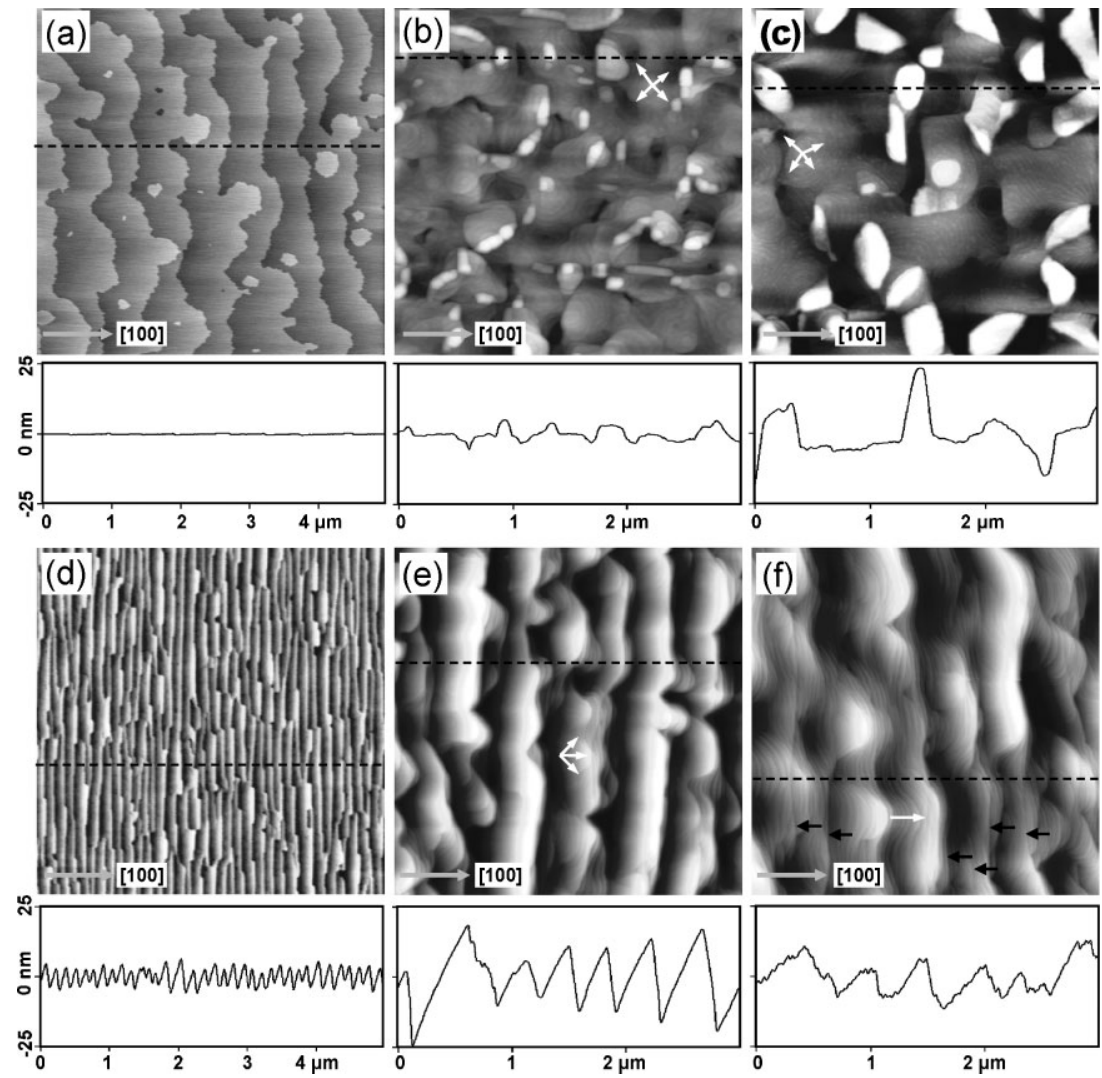

Figure 1. AFM images of a,d) before and after b,e) $100 \mathrm{~nm}$ and c,f) $400 \mathrm{~nm}$ thick $\mathrm{BiFeO}_{3}$ films on a-c) exact and d-f) miscut $\mathrm{SrTiO}_{3}$, with coherent $100 \mathrm{~nm}$ thick $\mathrm{SrRuO}_{3}$ bottom electrodes. The white arrows indicate the directions of step propagation. The line profiles were obtained across the dotted lines in the corresponding images. The black arrows in $f$ ) indicate domain boundaries.

estimated value based on the miscut angle $(6 \mathrm{~nm})$, due to step bunching. After the deposition of $\mathrm{BiFeO}_{3}$, the $100 \mathrm{~nm}$ thick $\mathrm{BiFeO}_{3}$ film displays terraces $500 \mathrm{~nm}$ wide (Fig. 1e), which means that step bunching also happens during the growth of $\mathrm{BiFeO}_{3}$ on the miscut substrate. The atomic steps propagate along the miscut direction. As the film gets thicker, the film surface gets smoother, and the flat terraces fade away with the formation of a number of small steps along the miscut direction (Fig. 1f). Note that the thicker film still exhibits a surface morphology of the step-flow growth mode ${ }^{[11]}$ without formation of $3 \mathrm{D}$ islands. The step-flow growth of $\mathrm{BiFeO}_{3}$ was also reported using orthorhombic $\mathrm{DyScO}_{3}$ substrates. ${ }^{[9]}$

In order to determine the domain structures and the crystallographic distortion of each domain with respect to the substrate miscut direction, $\mathrm{BiFeO}_{3}$ films were investigated using the HRXRD RSM technique. Figure 2 shows RSM patterns around $\mathrm{SrTiO}_{3} 013$ reflections for $\mathrm{BiFeO}_{3}$ films $400 \mathrm{~nm}$ thick on exact and miscut $\mathrm{SrTiO}_{3}$. The single narrow peak for $\mathrm{SrRuO}_{3} 013_{\mathrm{p}}$ reflections indicates that the $\mathrm{SrRuO}_{3}$ layers on both substrates are single-domain. Since the crystal structure of $\mathrm{SrRuO}_{3}$ is cubic at the $\mathrm{BiFeO}_{3}$ growth temperature $\left(690^{\circ} \mathrm{C}\right),{ }^{[12]}$ the surface of the $\mathrm{SrRuO}_{3}$ layer is crystallographically identical to that of the underlying $\mathrm{SrTiO}_{3}$ substrate. This indicates that the $\mathrm{SrRuO}_{3}$ bottom electrodes have no additional effect on the growth of
$\mathrm{BiFeO}_{3}$. In contrast to the $\mathrm{SrRuO}_{3}$ bottom electrodes, $\mathrm{BiFeO}_{3}$ films show broad peaks with different shapes compared with the substrate. Analysis on the RSM patterns suggested that the $\mathrm{BiFeO}_{3}$ film on exact $\mathrm{SrTiO}_{3}$ has four domains (Fig. 2a), but clearly exhibits only two domains on miscut $\mathrm{SrTiO}_{3}$ (Fig. 2c). The $\mathrm{BiFeO}_{3}$ film on exact $\mathrm{SrTiO}_{3}$ shows the same intensity for each domain, whereas that on miscut $\mathrm{SrTiO}_{3}$ displays two different peak intensities. The RSM pattern around the $0 \overline{3} 3 \mathrm{SrTiO}_{3}$ reflection (not shown here) revealed that the peak intensities of both $\mathrm{BiFeO}_{3}$ domains on miscut $\mathrm{SrTiO}_{3}$ are exactly reversed after rotating the film by $\Phi=180^{\circ}$, indicating the equal amount of both domains in the film. According to Streiffer et al., ${ }^{[6]}$ there are four structural domains of a rhombohedral phase, $r_{1}, r_{2}, r_{3}$, and $r_{4}$. Using these notations, we can identify all domains of both $\mathrm{BiFeO}_{3}$ films, as shown in Figure $2 \mathrm{~b}$ and d. Note that the $\mathrm{BiFeO}_{3}$ film on miscut (001) $\mathrm{SrTiO}_{3}$ has the rhombohedral distortion only along the [100] direction (distortion angle $\alpha_{\mathrm{m}}=0.65^{\circ}$ ), while that on exact $\mathrm{SrTiO}_{3}$ has the distortion along both $[100]$ and $[\overline{100}]$ directions $\left(\alpha_{\mathrm{e}}= \pm 0.4^{\circ}\right)$. These results provide direct evidence that the miscut substrate significantly affects the domain structure of the $\mathrm{BiFeO}_{3}$ film. The peak width of $\mathrm{BiFeO}_{3}$ film along the $0 k 0$ direction is much narrower in the film on miscut $\mathrm{SrTiO}_{3}$, indicating the improvement of crystalline quality in $\mathrm{BiFeO}_{3}$ films by using that substrate. Details on the crystal symmetry and domain structure of the $\mathrm{BiFeO}_{3}$ films on exact and miscut (001) $\mathrm{SrTiO}_{3}$ substrates will be discussed elsewhere. ${ }^{[10]}$

In the cross-sectional views of a (001) rhombohedral film along [100] direction by Streiffer et al., ${ }^{[6]} r_{1} / r_{2}$ or $r_{3} / r_{4}$ pairs form $\{100\}$ twin boundaries, and $r_{1} / r_{4}$ or $r_{2} / r_{3}$ pairs form $\{101\}$ twin boundaries. It suggests that a film with all four $r_{1}, r_{2}, r_{3}$, and $r_{4}$ domains can have both $\{100\}$ and $\{101\}$ twin boundaries, but a film with only two domains will have one preferred boundary orientation. This is consistent with our experimental results. Figure 3a and c show cross-sectional TEM images of $600 \mathrm{~nm}$ thick $\mathrm{BiFeO}_{3}$ films on exact and miscut (001) $\mathrm{SrTiO}_{3}$, respectively. The film on the exact substrate shows irregular domains with both $\{100\}$ and $\{101\}$ twin boundaries. In contrast, the film on the miscut substrate exhibits periodic domains with $\{101\}$ twin boundaries, namely stripe domains. Corresponding domain configurations for both films are identified and schematically presented. The width of stripe domains is determined to be $200 \sim 250 \mathrm{~nm}$ for $400 \sim 600 \mathrm{~nm}$ films, ${ }^{[13]}$ which is consistent with the spacing between domain boundaries parallel with the nonmiscut direction seen in the AFM image in Figure 1f. It is clear that the $\mathrm{BiFeO}_{3}$ film on the exact substrate has four polarization variants (Fig. 3a), and the $\mathrm{BiFeO}_{3}$ film on the miscut substrate has two polarization variants (Fig. 3c). The in-plane PFM images confirm four variants in the 

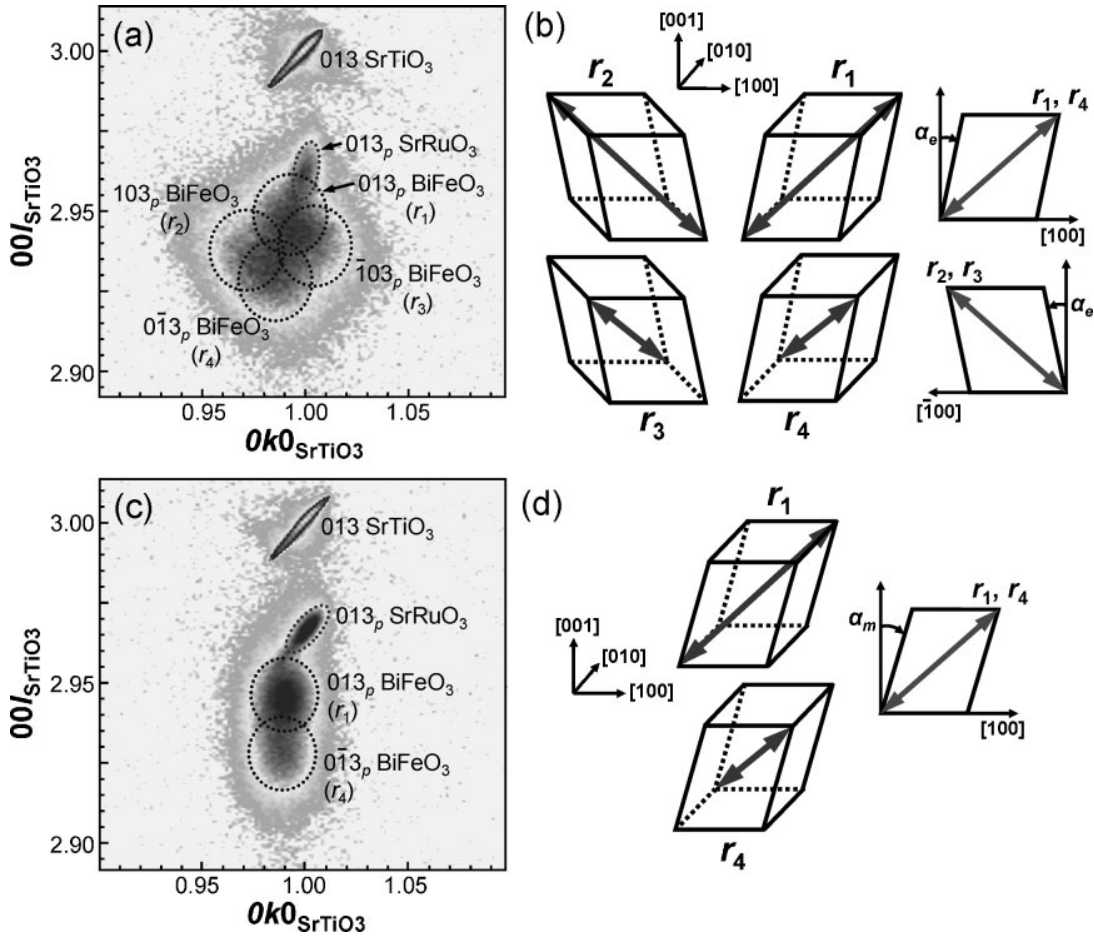

(d)

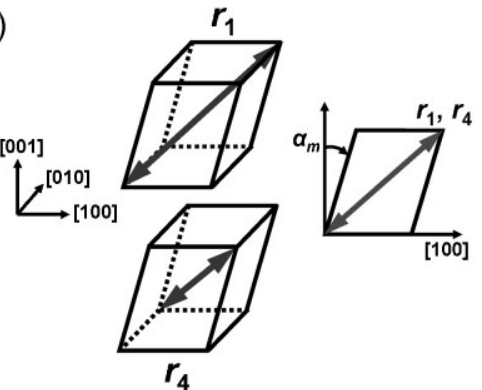

Figure 2. RSM images around $103 \mathrm{SrTiO}_{3}$ reflections for $\mathrm{BiFeO}_{3}$ films $400 \mathrm{~nm}$ thick on a) exact and c) miscut $\mathrm{SrTiO}_{3}$. Subscript $p$ represents pseudocubic setting. The schematics describe b) four structural domains in the film on the exact substrate and d) two domains in the film on the miscut substrate. The black arrows indicate the polarization direction of each domain in the unit cell and the (010) plane. $\alpha_{\mathrm{e}}\left(=0.4^{\circ}\right)$ and $\alpha_{\mathrm{m}}\left(=0.65^{\circ}\right)$ are the rhombohedral distortion angles. (a)
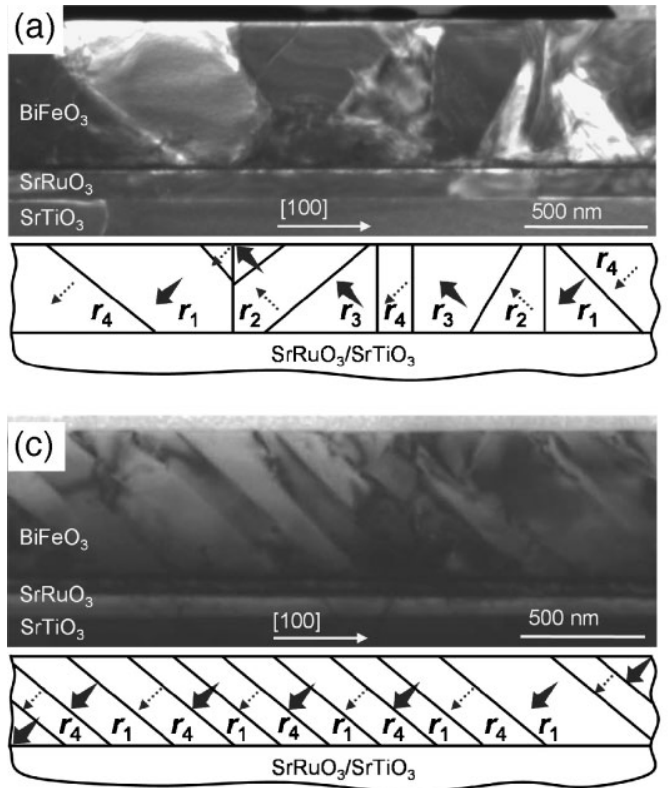
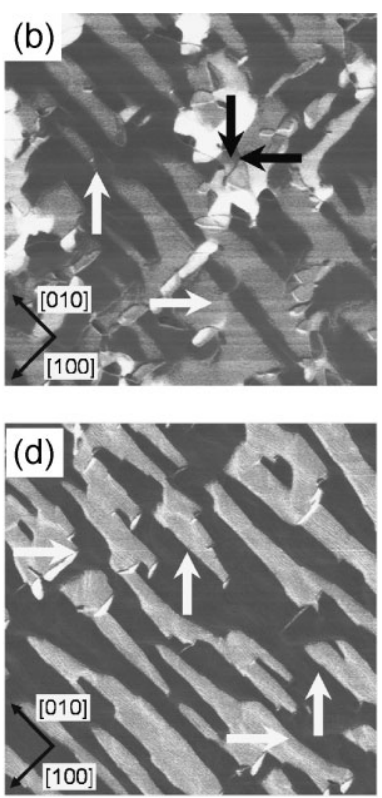

Figure 3. Cross-sectional TEM images of $\mathrm{BiFeO}_{3}$ films $600 \mathrm{~nm}$ thick on a) exact and c) miscut $\mathrm{SrTiO}_{3}$. In-plane PFM images ( $\left.4 \mu \mathrm{m} \times 4 \mu \mathrm{m}\right)$ of $\mathrm{BiFeO}_{3}$ films $40 \mathrm{~nm}$ thick on b) exact and d) miscut $\mathrm{SrTiO}_{3}$ were obtained with an ac bias of $3 \mathrm{~V}_{\mathrm{pp}}$ at $6.39 \mathrm{kHz}$. The arrows in each PFM image represent directions of in-plane polarization, indicating four variants on exact $\mathrm{SrTiO}_{3}$ and two variants on miscut $\mathrm{SrTiO}_{3}$
$\mathrm{BiFeO}_{3}$ film on exact $\mathrm{SrTiO}_{3}$ (Fig. 3b) and two variants in the $\mathrm{BiFeO}_{3}$ film on miscut $\mathrm{SrTiO}_{3}$ (Fig. 3d).

It was observed that an $800 \mathrm{~nm}$ thick $\mathrm{BiFeO}_{3}$ film on miscut substrate still has only two variants. This fact suggested that the elasticstrain energy in $\mathrm{BiFeO}_{3}$ films on miscut substrates are effectively relieved without forming additional domain variants $\left(r_{2}\right.$ and $\left.r_{3}\right)$. There are two competing mechanisms for strain relaxation, namely surface roughening and crystallographic tilt by dislocation multiplication. ${ }^{[14]}$ The AFM images clearly indicate that the $\mathrm{BiFeO}_{3}$ films on exact $\mathrm{SrTiO}_{3}$ exhibit strain relaxation by surface roughening. However, there was no surface roughening in the $\mathrm{BiFeO}_{3}$ films on miscut $\mathrm{SrTiO}_{3}$. Thus, we measured the crystallographic tilt of $\mathrm{BiFeO}_{3}$ films respective to the $\mathrm{SrRuO}_{3}$ bottom electrodes using RSM patterns around $002 \mathrm{SrTiO}_{3}$ reflections along two orthogonal directions (miscut and nonmiscut directions), as shown in Figure 4a. As expected, there is no film tilt along the [010] direction (nonmiscut direction) because the miscut angle does not exist for the films to tilt against the substrate. However, the tilt of the films along the [100] direction (miscut direction) is significant. The highly strained 20 and $50 \mathrm{~nm}$ films have negative tilt angles, due to the intrinsic tilt mechanism described by Nagai, ${ }^{[15]}$ in which a compressively strained coherent film tilts away from the surface normal due to lattice mismatch on the surface steps of the miscut substrate, as shown in the inset of Figure 4a. With increasing film thickness, the film tilts toward the direction normal to the surface, reducing the angle between this and the [001] direction of the film (Fig. 4b). This positive tilt is evidence of preferential dislocation nucleation, which corresponds to strain relaxation in the film to relieve its total elastic energy. ${ }^{[14]}$ It should be noted that the tilt of the $\mathrm{BiFeO}_{3}$ films is observed along the miscut direction, and not in the nonmiscut direction.

The tilting of $\mathrm{BiFeO}_{3}$ films along the [001] direction is attributed to the anisotropic strain relaxation in the films on miscut substrates. Figure $4 c$ and $d$ show the in-plane and out-ofplane lattice parameters of $\mathrm{BiFeO}_{3}$ films on exact and miscut $\mathrm{SrTiO}_{3}$ as a function of film thickness. With increasing film thickness, the in-plane lattice parameters increase and the out-of-lattice parameters decrease, due to the relaxation of biaxial compressive strains. As the film thickness increases, the films on exact $\mathrm{SrTiO}_{3}$ display almost identical variation in inplane lattice parameters along [100] and [010] directions (Fig. 4c). In contrast, the films on 


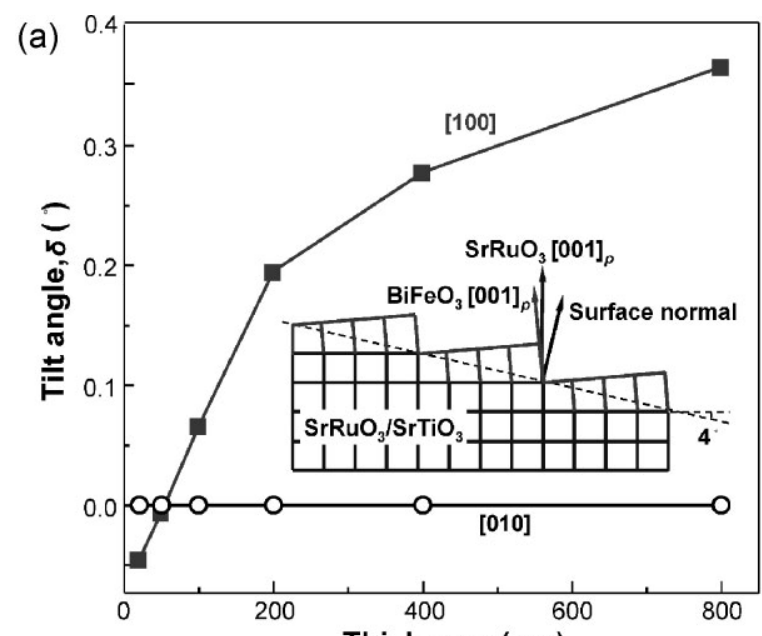

(c)
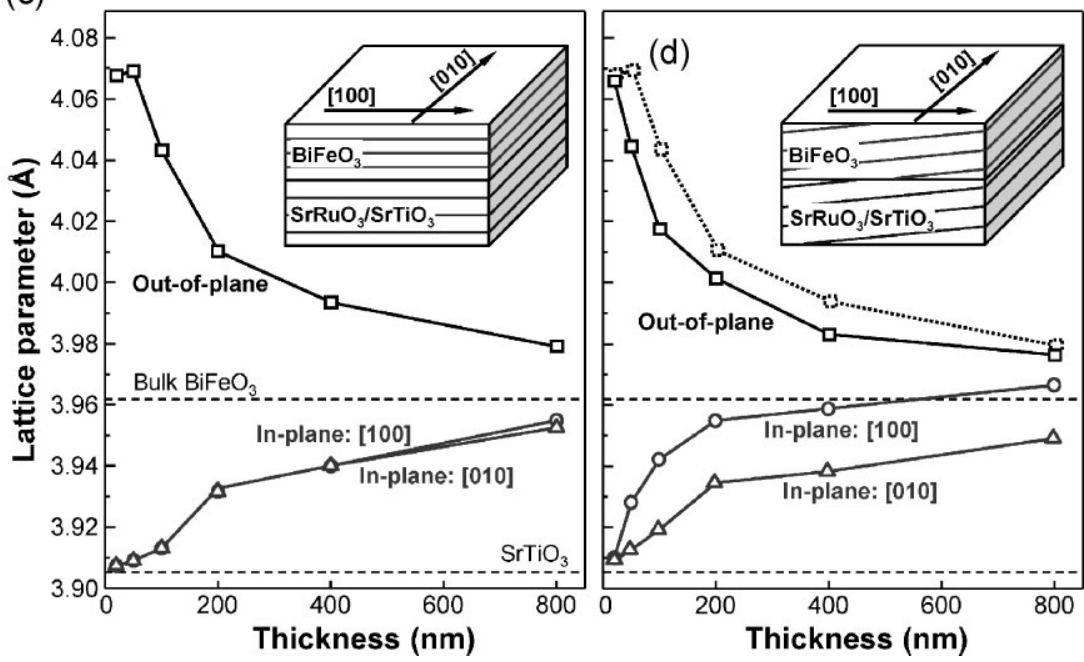

Figure 4. a) Tilt angle as a function of film thickness for $\mathrm{BiFeO}_{3}$ films on miscut $\mathrm{SrTiO}_{3}$ substrate with $\mathrm{SrRuO}_{3}$ bottom electrodes. The inset is a schematic for the negative tilting of coherent $\mathrm{BiFeO}_{3}$ films on the miscut $\mathrm{SrRuO}_{3} / \mathrm{SrTiO}_{3}$ substrate. b) Schematic diagrams for the change of the tilt angle for the $\mathrm{BiFeO}_{3}$ films with thickness. Pseudocubic lattice parameters were measured as a function of film thickness for $\mathrm{BiFeO}_{3}$ films grown on c) exact and d) miscut $\mathrm{SrTiO}_{3}$. The dotted line in d) represents the out-of-plane lattice parameter of the $\mathrm{BiFeO}_{3}$ film exact $\mathrm{SrTiO}_{3}$.

miscut $\mathrm{SrTiO}_{3}$ show faster in-plane strain relaxation along the miscut direction ([100] direction) than along the nonmiscut direction ([010] direction) (Fig. 4d). Thus, the overall strain relaxation is faster in the films on miscut $\mathrm{SrTiO}_{3}$. This result confirms the in-plane anisotropy of the miscut substrate, which has also been observed in the tilting of the crystallographic planes and in the AFM images.

Based on these experimental results, the origin of ferroelastic domain variant selection in epitaxial (001) $\mathrm{BiFeO}_{3}$ films by the miscut substrate is described as follows. On the exact substrate, the terrace width is too large for $\mathrm{BiFeO}_{3}$ to grow in a step-flow growth mode. As a result, 3D islands are formed. As the film gets thicker, the shear strain in the film, induced by the rhombohedral symmetry of $\mathrm{BiFeO}_{3}$, should be relieved by the rhombohedral distortion of the unit cells. Since the film has biaxially isotropic strain, the rhombohedral distortion occurs randomly, and is described as distortions along both [100] and [100] directions in the (010) plane. As seen in Figure $5 \mathrm{a}, r_{1}$ and $r_{4}$ are twins with the (101) plane, and $r_{1}$ and $r_{2}$ with the (100) plane. As a result, all four $r_{1}, r_{2}, r_{3}$, and $r_{4}$ domains are formed in the film $\{101\}$ and $\{100\}$ twin boundaries. It is easily speculated that $r_{2}$ or $r_{3}$ domains are formed preferentially to the 3D islands, creating $\{100\}$ boundaries. On the miscut substrate, the small terrace width drives $\mathrm{BiFeO}_{3}$ to adopting a complete stepflow growth mode. This prevents the formation of 3D islands for $r_{2}$ or $r_{3}$ domains, because the rhombohedral distortion toward the step edge is not energetically favorable, as shown in Figure 5b. Furthermore, the miscut substrate drives the films to tilting along the [100] direction (Fig. 4a), which corresponds to the preferential rhombohedral distortions to the downhill miscut direction, as shown in Figure $5 \mathrm{~b}$. In other words, the miscut substrate removes from the film the degree of freedom of having rhombohedral distortions for $r_{2}$ and $r_{3}$. Consequently, $r_{1}$ and $r_{4}$ stripe domains with (101) twin boundaries are formed in the film.

The effects on the ferroelectric properties of $\mathrm{BiFeO}_{3}$ films can be explored by measuring $P-E$ hysteresis loops. The domain selection by the miscut substrate greatly affects the ferroelectric switching behavior of $\mathrm{BiFeO}_{3}$. The $400 \mathrm{~nm}$ thick $\mathrm{BiFeO}_{3}$ film on miscut $\mathrm{SrTiO}_{3}$ exhibits perfect square-like $P-E$ loops (Fig. 6a). The remanent polarization $\left(P_{\mathrm{r}}\right)$ value is measured to be $64 \pm 2 \mu \mathrm{C} \mathrm{cm}^{-2}$. Compared with the $P_{\mathrm{r}}$ of bulk single-crystal $\mathrm{BiFeO}_{3}$ $\left(\sim 60 \mu \mathrm{C} \mathrm{cm}{ }^{-2}\right),{ }^{[16]}$ it is concluded that complete domain switching is obtained from the $\mathrm{BiFeO}_{3}$ films with the two-variant stripe domains. The higher $P_{\mathrm{r}}$ values of the films than those of $\mathrm{BiFeO}_{3}$ bulk single-crystal can be attributed to the strain-induced polarization rotation. ${ }^{[17]}$ In contrast, the $400 \mathrm{~nm}$ thick $\mathrm{BiFeO}_{3}$ film on exact $\mathrm{SrTiO}_{3}$ displays an unclosed and slanted loops at the lower frequency, and drastically lower $P_{\mathrm{r}}$ values of $43 \pm 5 \mu \mathrm{C} \mathrm{cm}^{-2}$ (Fig. 6b). The slanted loop indicates the nonuniformity of the domains, in that each one in the film has a different coercivity. This suggests that some of domains are very hard to switch with applied field, leading to the lower $P_{r}$. As seen in TEM and PFM images, more uniform and ordered ferroelectric (ferroelastic) domains are seen for films on miscut than on exact substrates, contributing to a square-like loop rather than a slanted $P-E$ loop. To confirm the significantly different switching behaviors between both films, pulsed-polarization measurements were carried out as functions of applied field and pulse width. Figure $6 \mathrm{c}$ shows the switching polarization $(\Delta P)$ as a function of electric field. The saturated $\Delta P$ values for both films are in excellent agreement with the $2 P_{\mathrm{r}}$ value from the $P-E$ loops, clarifying the reduced $P_{\mathrm{r}}$ in the film on exact $\mathrm{SrTiO}_{3}$. From 

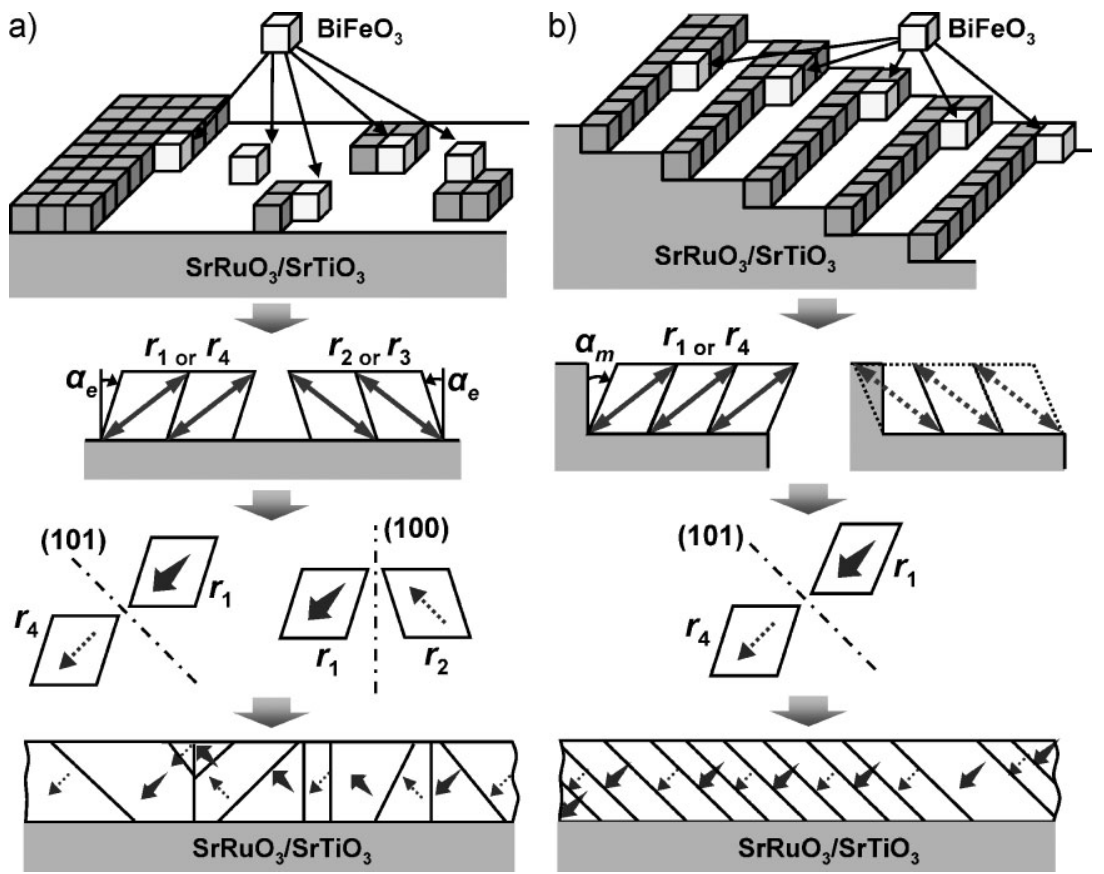

Figure 5. Schematic drawings for the initial growth, domain selection, formation of domain boundaries, and final structure for $\mathrm{BiFeO}_{3}$ films on a) exact and b) miscut $\mathrm{SrTiO}_{3}$ substrates with $\mathrm{SrRuO}_{3}$ bottom electrodes. The formation of $r_{2}$ and $r_{3}$ domains is prohibited in $\mathrm{BiFeO}_{3}$ films on miscut $\mathrm{SrTiO}_{3}$ substrates, because the rhombohedral distortion for $r_{2}$ and $r_{3}$ is mechanically unfavorable. (a)

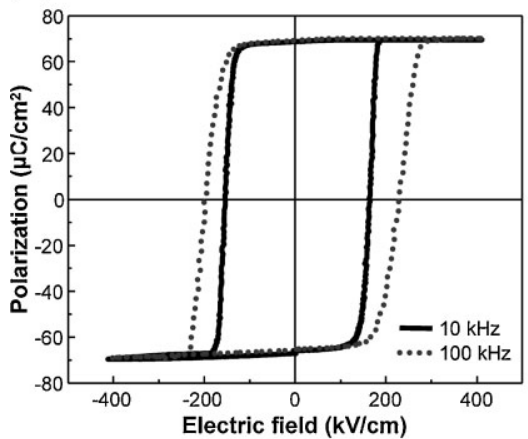

(c)

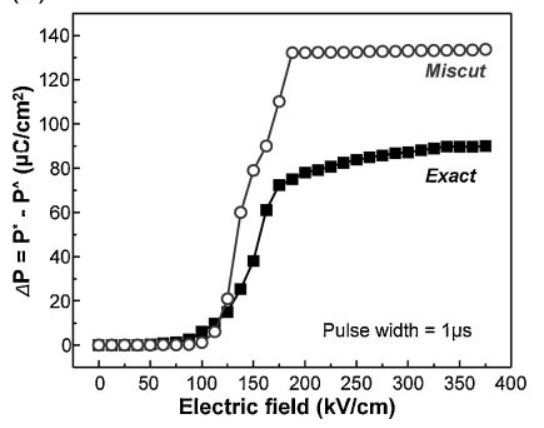

(b)

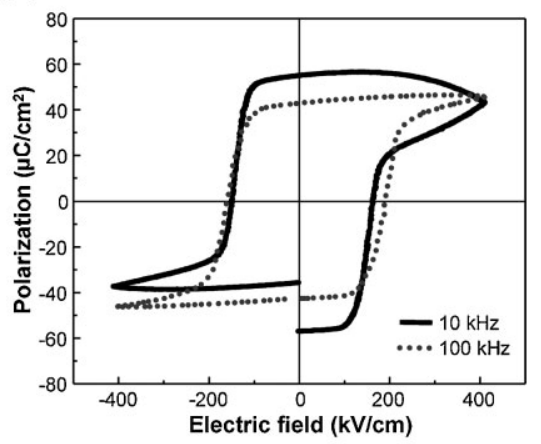

(d)

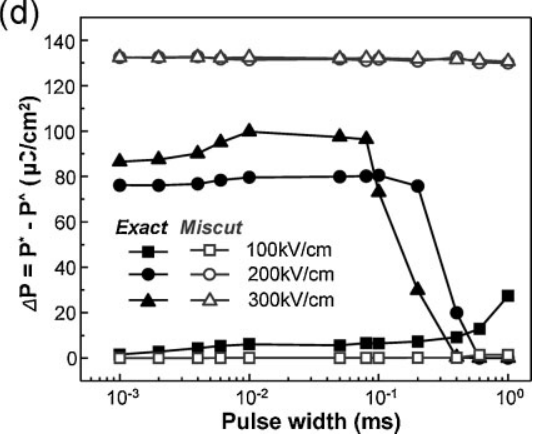

Figure 6. $P-E$ hysteresis loops of $\mathrm{BiFeO}_{3}$ films $400 \mathrm{~nm}$ thick on a) miscut and b) exact $\mathrm{SrTiO}_{3}$ at room temperature. Switching polarization $(\Delta P)$ as a function of c) applied electric field and d) pulse width for $\mathrm{BiFeO}_{3}$ films $400 \mathrm{~nm}$ thick on miscut and exact $\mathrm{SrTiO}_{3}$. starting to increase to saturating, the $\Delta P$ of the film on miscut $\mathrm{SrTiO}_{3}$ shows a narrower and abrupt transition, whereas that of the film on the exact $\mathrm{SrTiO}_{3}$ shows a more gradual transition, consistent with the difference in the shape of $P-E$ loops (square-like vs. slanted). With increasing the pulse width from $1 \mu$ s to 1 ms, the $\Delta P$ of the film on miscut $\mathrm{SrTiO}_{3}$ remained constant at $132 \mu \mathrm{C} \mathrm{cm}^{-2}$ at 200 and $300 \mathrm{kV} \mathrm{m}^{-1}$. However, the film on exact $\mathrm{SrTiO}_{3}$ shows different $\Delta P$ values at 200 and $300 \mathrm{kV}$ $\mathrm{m}^{-1}$, and a stark contrast when $\Delta P$ becomes zero with pulses approaching $1 \mathrm{~ms}$ at 200 and $300 \mathrm{kV} \mathrm{m}^{-1}$. This means that the film does not switch at all with long pulse widths.

Significant reduction in leakage current density is found in $\mathrm{BiFeO}_{3}$ films on miscut $\mathrm{SrTiO}_{3}$. Figure 7a shows leakage current density as a function of applied electric field for $400 \mathrm{~nm}$ thick $\mathrm{BiFeO}_{3}$ films on both exact and miscut $\mathrm{SrTiO}_{3}$. The film on the exact substrate displays a leakage current density around $2 \times 10^{-3} \mathrm{~A} \mathrm{~cm}^{-2}$ at $100 \mathrm{kV} \mathrm{cm}^{-1}$, which is comparable to the previously reported values between $5 \times 10^{-1}$ and $5 \times 10^{-3} \mathrm{~A} \mathrm{~cm}^{-2}$ at $100 \mathrm{kV} \mathrm{cm}{ }^{-1} \cdot{ }^{[18-20]}$ Note that the leakage current density is reduced by two orders of magnitude for the film on the miscut substrate. The leakage current density of $3 \times 10^{-5} \mathrm{~A}$ $\mathrm{cm}^{-2}$ at $100 \mathrm{kV} \mathrm{cm}^{-1}$ is the lowest value ever reported for $\mathrm{BiFeO}_{3}$ films. ${ }^{[21]}$ Current-time measurements were carried out to examine current relaxation in both films, as shown in Figure $7 \mathrm{~b}$. The leakage current for the film on the miscut substrate stabilizes in $10 \mathrm{~ms}$, whereas the current relaxation for the film on the exact substrate is very slow, and does not stabilize even in $100 \mathrm{~ms}$, implying that the film has a lot of free charge carriers with oxygen vacancies, resulting in high leakage current levels and slow current relaxation times. ${ }^{[22]}$

We believe there are two possible mechanisms for the origin of the higher leakage current in $\mathrm{BiFeO}_{3}$ films on exact $\mathrm{SrTiO}_{3}$, namely i) domain structure and ii) nonstoichiometric point defects. The first mechanism operates through the notion that $109^{\circ}$ domain walls are dominant leakage paths rather than the bulk matrix, as shown in Figure 7c. According to Streiffer et al., ${ }^{[6]}$ a rotational deformation of opposite sense about the (010) direction in adjacent domains is required to bond the vertical $109^{\circ}$ domains to the substrate, which is not necessary for the $71^{\circ}$ domains with (101) twin boundaries to be bonded to the substrate. This additional deformation can cause the width of the $109^{\circ}$ domain walls to be larger and generate charge carriers near the walls. In addition, the 109 

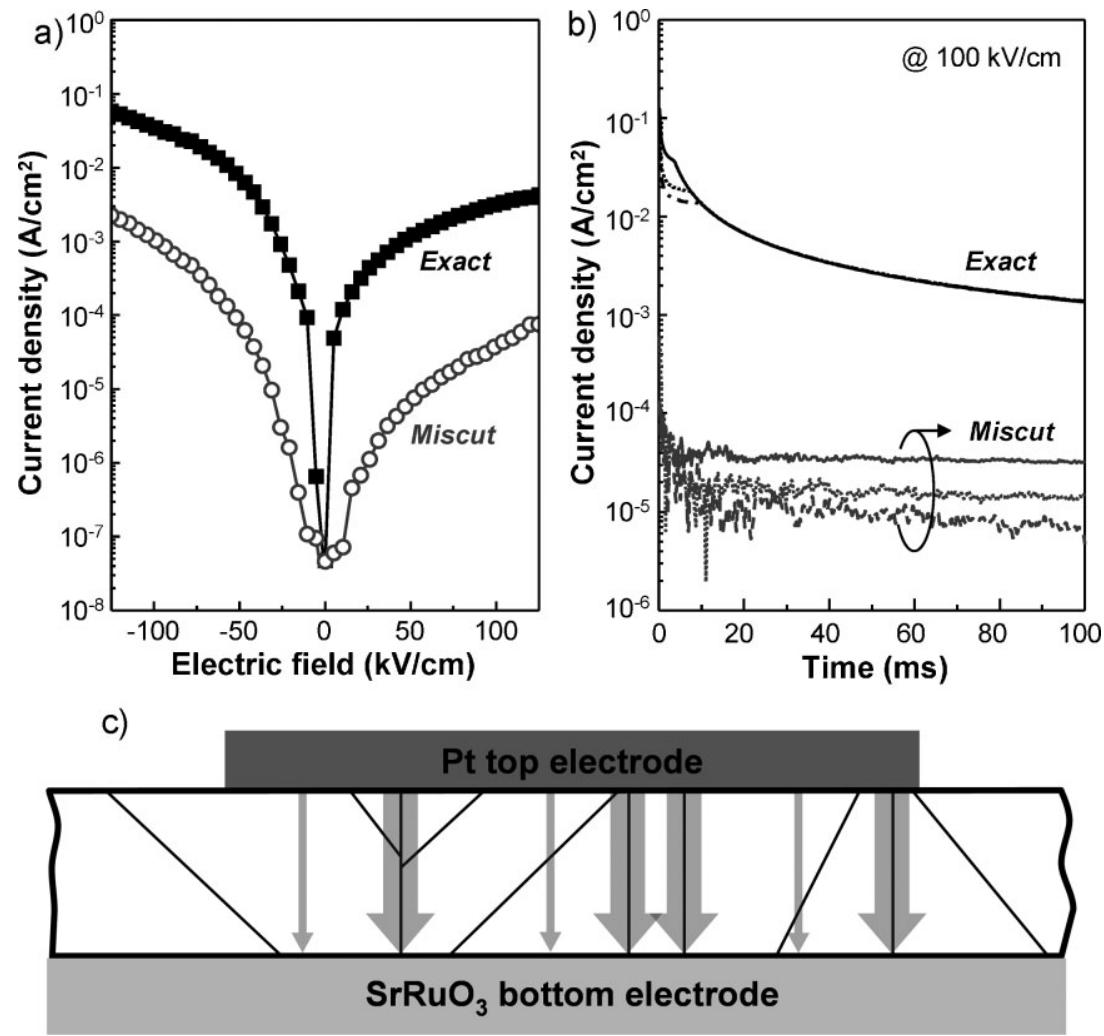

Figure 7. a) Current-voltage characteristics of $\mathrm{BiFeO}_{3}$ films $400 \mathrm{~nm}$ thick on exact and miscut $\mathrm{SrTiO}_{3}$. The measure time for each point was $100 \mathrm{~ms}$. b) Current-time characteristics of $\mathrm{BiFeO}_{3}$ films $400 \mathrm{~nm}$ thick on exact and miscut $\mathrm{SrTiO}_{3}$. c) Schematic drawing for leakage paths in $\mathrm{BiFeO}_{3}$ film $400 \mathrm{~nm}$ thick on exact $\mathrm{SrTiO}_{3}$. The arrows represent leakage current through the film, showing that the vertical $109^{\circ}$ domain walls are dominant leakage paths rather than the bulk matrix.
$\mathrm{BiFeO}_{3}$ films on exact substrates. The domainwall motions for polarization switching can be delayed and impeded by the larger walls between $109^{\circ}$ domains, resulting in incomplete switching with the applied electric filed. At very high electric fields, nonswitchable domains can be switched, and then complete switching can be obtained. However, at such high fields, the leakage current through the vertical $109^{\circ}$ domain walls and/or bulk matrices becomes predominant, and thus complete switching cannot be obtained. For this same reason, the films do not switch with the long pulse widths in Figure 6d. A recent study on PFM of (011) $\mathrm{BiFeO}_{3}$ films showed that $109^{\circ}$ domain switching is less stable and less electrically controllable than $71^{\circ}$ domain switching, partially supporting our suggestion. ${ }^{[25]}$

In conclusion, we have demonstrated the selection of domain-structure variants in epitaxial $\mathrm{BiFeO}_{3}$ films, and consequently achieved significant improvement in ferroelectric switching behavior and leakage current by employing miscut in cubic (001) $\mathrm{SrTiO}_{3}$ substrates. $\mathrm{BiFeO}_{3}$ films on miscut $\mathrm{SrTiO}_{3}$ have a step-flow growth and a preferential rhombohedral distortion toward the miscut direction. This result indicates that both the substrate anisotropy and the step-flow growth of $\mathrm{BiFeO}_{3}$ are the origins of the two-variant stripe domains in $\mathrm{BiFeO}_{3}$ films. Square-like $P-E$ loops and very low leakage current densities are obtained from these $\mathrm{BiFeO}_{3}$ films. In domain wall is parallel to the electric field. Thus, the $109^{\circ}$ domain wall can become a predominant leakage path in the film on exact $\mathrm{SrTiO}_{3}$, leading to the high leakage current.

The second mechanism operates through the notion that the bulk matrix is the dominant leakage path rather than domain walls. Although $\mathrm{BiFeO}_{3}$ films on exact as well as miscut $\mathrm{SrTiO}_{3}$ are stoichiometric, with a $\mathrm{Bi} / \mathrm{Fe}$ 1:1 ratio, and have no secondary phases, there is the possibility of formation of point defects, such as oxygen vacancies, in the films. It is generally accepted that the miscut substrate leads to the formation of preferential domains and the stabilization of stoichiometric phases. ${ }^{[23]}$ The longer step width on exact $\mathrm{SrTiO}_{3}$ means longer time for adapted atoms to reach the step edge and crystallize. The relatively volatile bismuth adatoms can evaporate from the film surface during the growth, and thus bismuth and oxygen vacancies are formed in the films after growth. As a result, those nonstoichiometric point defects spread out in the films, leading to the large leakage current. Detailed studies on the high leakage current on exact $\mathrm{SrTiO}_{3}$ are currently underway.

In epitaxial (001) $\mathrm{BiFeO}_{3}$ films, polarization switching occurs with the formation of both ferroelectric and ferroelastic domain walls. ${ }^{[7]}$ According to Wicks et al., ${ }^{[24]}$ grain boundaries in $\mathrm{Pb}\left(\mathrm{Zr}_{x} \mathrm{Ti}_{1-x}\right) \mathrm{O}_{3}$ thin films impede domain-wall movement, and are able to nucleate domains that are opposite to those generated by an electric field. From this, we believe that $109^{\circ}$ domain walls contribute to the reduction in $P_{\mathrm{r}}$ due to incomplete switching for contrast, $\mathrm{BiFeO}_{3}$ films on exact $\mathrm{SrTiO}_{3}$ exhibit low $P_{\mathrm{r}}$ values and high leakage currents. From this, we suggest that $109^{\circ}$ domain walls prevent the complete ferroelectric switching of (001) $\mathrm{BiFeO}_{3}$ domains and act as dominant leakage paths in the four-variant $\mathrm{BiFeO}_{3}$ films, whereas the intrinsic ferroelectric properties of $\mathrm{BiFeO}_{3}$ can be observed from two-variant $\mathrm{BiFeO}_{3}$ films with $71^{\circ}$ domain walls. ${ }^{[26]}$ The dependence of ferroelectric properties on domain-wall configuration opens the exciting opportunity to investigate the correlation between domain walls and the antiferromagnetic order in $\mathrm{BiFeO}_{3}$. We believe that such domain engineering can be very useful for growing high-quality $\mathrm{BiFeO}_{3}$ films on cubic (001) Si substrates for device applications ${ }^{[27]}$ and more generally for heterostructures with rhombohedral thin films, such as $\mathrm{Pb}\left(\mathrm{Zr}_{x} \mathrm{Ti}_{1-x}\right) \mathrm{O}_{3}, \mathrm{~Pb}\left(\mathrm{Mg}_{1 / 3} \mathrm{Nb}_{2 / 3}\right) \mathrm{O}_{3}-\mathrm{PbTiO}_{3}$, $\mathrm{La}_{1-x} \mathrm{Sr}_{x} \mathrm{MnO}_{3}$, and $\mathrm{LaAlO}_{3}$.

\section{Experimental}

Epitaxial (001) $\mathrm{BiFeO}_{3}$ films were grown by off-axis radio-frequency ( $\mathrm{rf}$ ) magnetron sputtering on 0.05 and $4^{\circ}$ miscut $(001) \mathrm{SrTiO}_{3}$ substrates[8]. Prior to the deposition of the $\mathrm{BiFeO}_{3}$ films, an epitaxial $100 \mathrm{~nm}$ thick $\mathrm{SrRuO}_{3}$ bottom electrode was deposited by $90^{\circ}$ off-axis if magnetron sputtering [28]. The thicknesses of the $\mathrm{BiFeO}_{3}$ films were varied from 20 to $800 \mathrm{~nm}$. The surface morphology and piezoelectric properties of $\mathrm{BiFeO}_{3} /$ $\mathrm{SrRuO}_{3}$ heterostructures were investigated using a DI Multimode AFM system [7]. A commercially available high-resolution four-circle $\mathrm{X}$-ray 
diffractometer (D8 Advance, Bruker $\mathrm{A} \times \mathrm{S}$ ) was used in HRXRD-RSM measurements. TEM studies were carried out on a Philips CM 12 operated at $120 \mathrm{kV}$ with a high-angle $\left( \pm 60^{\circ}\right)$ double-tilt holder, and on a JEOL 3011 ultrahigh-resolution TEM operated at $300 \mathrm{kV}$ with a point-to-point resolution of $0.17 \mathrm{~nm}$. Pt top electrodes $(100 \mu \mathrm{m}$ in diameter) were patterned to measure the ferroelectric properties using a Radiant PFH100 ferroelectric measurement system.

\section{Acknowledgements}

The authors gratefully acknowledge the financial support of the National Science Foundation through grants ECCS-0708759, 0425914, the Office of Naval Research through grant N00014-05-1-0559, and the Department of Energy under grant DE-FC02-07ER46416. The corresponding author wants to thank Paul Evans for helpful discussions.

Received: March 25, 2008 Revised: August 20, 2008

[1] N. A. Spaldin, M. Fiebig, Science 2005, 309, 391.

[2] W. Eerenstein, N. D. Mathur, J. F. Scott, Nature 2006, 442, 759.

[3] T. Zhao, A. Scholl, F. Zavaliche, K. Lee, M. Barry, A. Doran, M. P. Cruz, Y. H. Chu, C. Ederer, N. A. Spaldin, R. R. Das, D. M. Kim, S. H. Baek, C. B. Eom, R. Ramesh, Nat. Mater. 2006, 5, 823.

[4] R. Ramesh, N. A. Spaldin, Nat. Mater. 2007, 6, 21.

[5] F. Kubel, H. Schmid, Acta Crystallogr. Sect. B 1990, 46, 698.

[6] S. K. Streiffer, C. B. Parker, A. E. Romanov, M. J. Lefevre, L. Zhao, J. S. Speck, W. Pompe, C. M. Foster, G. R. Bai, J. Appl. Phys. 1998, 83, 2742.

[7] F. Zavaliche, P. Shafer, R. Ramesh, M. P. Cruz, R. R. Das, D. M. Kim, C. B. Eom, Appl. Phys. Lett. 2005, 87, 252902.

[8] R. R. Das, D. M. Kim, S. H. Baek, C. B. Eom, F. Zavaliche, S. Y. Yang, R. Ramesh, Y. B. Chen, X. Q. Pan, X. Ke, M. S. Rzchowski, S. K. Streiffer, Appl. Phys. Lett. 2006, 88, 242904.

[9] Y. H. Chu, Q. Zhan, L. W. Martin, M. P. Cruz, P. L. Yang, G. W. Pabst, F. Zavaliche, S. Y. Yang, J. X. Zhang, L. Q. Chen, D. G. Schlom, T. B. Wu, R. Ramesh, Adv. Mater. 2006, 18, 2307.
[10] C. M. Folkman, H. W. Jang, D. Ortiz, S. H. Baek, C. B. Eom, unpublished.

[11] M. D. Johnson, C. Orme, A. W. Hunt, D. Graff, J. Sudijono, L. M. Sander, B. G. Orr, Phys. Rev. Lett. 1994, 72, 116.

[12] J. P. Maria, H. L. McKinstry, S. Trolier-McKinstry, Appl. Phys. Lett. 2000, 76 , 3382.

[13] Y. B. Chen, M. B. Katz, X. Q. Pan, R. R. Das, D. M. Kim, S. H. Baek, C. B. Eom, Appl. Phys. Lett. 2007, 90, 072907.

[14] P. M. Mooney, Mater. Sci. Eng. R 1996, 17, 105.

[15] H. Nagai, J. Appl. Phys. 1972, 43, 4254.

[16] D. Lebeugle, D. Colson, A. Forget, M. Viret, P. Bonville, J. F. Marucco, S. Fusil, Phys. Rev. B 2007, 76, 024116.

[17] H. W. Jang, S. H. Baek, D. Ortiz, C. M. Folkman, R. R. Das, Y. H. Chu, P. Shafer, J. X. Zhang, S. Choudhury, V. Vaithyanathan, Y. B. Chen, D. A. Felker, M. D. Biegalski, M. S. Rzchowski, X. Q. Pan, D. G. Schlom, L. Q. Chen, R. Ramesh, C. B. Eom, Phys. Rev. Lett. 2008, 101, 107602.

[18] X. Qi, J. Dho, R. Tomov, M. G. Blamire, J. L. MacManus-Driscoll, Appl. Phys. Lett. 2005, 86, 062903.

[19] Y. Wang, C. W. Nan, Appl. Phys. Lett. 2006, 89, 052903.

[20] G. W. Pabst, L. W. Martin, Y. H. Chu, R. Ramesh, Appl. Phys. Lett. 2007, 90 072902.

[21] H. Yang, M. Jain, N. A. Suvorova, H. Zhou, H. M. Luo, D. M. Feldmann, P. C. Dowden, R. F. DePaula, S. R. Foltyn, Q. X. Jia, Appl. Phys. Lett. 2007, 91, 072911.

[22] B. Nagaraj, S. Aggarwal, R. Ramesh, J. Appl. Phys. 2001, 90, 375.

[23] S. D. Bu, M. K. Lee, C. B. Eom, W. Tian, X. Q. Pan, S. K. Streiffer, J. J. Krajewski, Appl. Phys. Lett. 2001, 79, 3482.

[24] S. Wicks, V. Anbusathiah, V. Nagarajan, Nanotechnology 2007, 18, 465502.

[25] H. W. Jang, S. H. Baek, D. Ortiz, C. M. Folkman, C. B. Eom, Y. H. Chu, P. Shafer, R. Ramesh, V. Vaithyanathan, D. G. Schlom, Appl. Phys. Lett. 2008, 92, 062910.

[26] M. P. Cruz, Y. H. Chu, J. X. Zhang, P. L. Yang, F. Zavaliche, Q. He, P. Shafer, L. Q. Chen, R. Ramesh, Phys. Rev. Lett. 2007, 99, 217601.

[27] S. H. Baek, R. R. Das, D. M. Kim, H. W. Jang, C. B. Eom, D. G. Schlom, Y. B. Chen, X. Q. Pan, F. Zavaliche, S. Y. Yang, R. Ramesh, unpublished.

[28] C. B. Eom, R. J. Cava, R. M. Fleming, J. M. Phillips, R. B. Vandover J. H. Marshall, J. W. P. Hsu, J. J. Krajewski, W. F. Peck, Science 1992, 258, 1766. 